\title{
No Joint Ownership! Shared Emotions Are Social-relational Emotions
}

\author{
Vivian Bohl \\ Department of Philosophy, University of Tartu
}

\begin{abstract}
There are cases of emotion that we readily describe as 'sharing emotions with other people.' How should we understand such cases? Joel Krueger has proposed the Joint Ownership Thesis (JOT): the view that two or more people can literally share the same emotional episode. His view is partly inspired by his reading of MerleauPonty-arguably Merleau-Ponty advocates a version of JOT in his “The child's relations with others." My critical analysis demonstrates that JOT is flawed in several respects: 1) It involves a vague account of joint subjects; 2) It relies on a confusion between phenomenological and ontological levels of analysis. When these are clearly distinguished, Krueger's phenomenological analysis contradicts JOT understood as an ontological claim; 3) It relies on a highly problematic coupling-constitution inference; 4) It relies on a shift from the claim that the child and the caregiver jointly realize an emotion, to the claim about joint ownership, which is a non sequitur. I argue that we can reach a better understanding of the phenomenon of shared emotions by bringing in another level of analysis: that of social relationships. I propose that shared emotions are a special case of social-relational emotions, typically arising within and/or giving rise to communal relationships.
\end{abstract}

Keywords: social cognition, social relationships, relational models theory, shared mental states, collective intentionality, joint ownership thesis

\section{Introduction}

'Emotion' is a common term in folk psychology as well as in scientific and philosophical research. In everyday language, people often talk about sharing emotions and other mental states. For example, we say that we share joy or sadness with a friend or a family member. But can we literally share affective states with others and, if we can do so, what is the proper concep-

Corresponding author's address: Vivian Bohl, Department of Philosophy, Institute of Philosophy and Semiotics, University of Tartu, Ülikooli 18, 50090 Tartu, Estonia. Email: vivian.bohl@ut.ee. 
tion of such states? Joel Krueger (2013) argues, proceeding from a reading of Maurice Merleau-Ponty ${ }^{1}$ (1964) and from an analysis of positive emotions in very young infants, that two individuals can literally own the same token of an emotion. He labels this claim the joint ownership thesis (JOT). ${ }^{2}$ In this paper, I argue against Krueger's JOT and propose that shared emotions are best conceptualized as a specific type of social-relational emotions.

I begin by outlining some of the most common ways philosophers have been thinking about collective or shared mental states (section 2). This will serve as a background for introducing Krueger's radical thesis-JOT-in section 3. In section 4, I argue against Krueger's account of JOT by showing that: 1) it fails to clearly distinguish between the thesis of joint ownership and the thesis of joint subjects and includes a vague account of joint subjects; 2) it lumps together phenomenological and ontological levels of analysis; when these two levels of analysis are kept apart, then the phenomenological analysis actually speaks against JOT as an ontological thesis; 3 ) it draws on a problematic inference from coupling to constitution; 4) it draws on a non sequitur inference from a claim about the realization of emotions to a claim about the ownership of emotions. Although JOT fails, from the perspective of social cognition research, the case of positive emotions in very young infants is still an interesting paradigm case of shared emotions. Positive emotions in infants seem to have some irreducibly intersubjective features that call for further exploration. In section 5 , I argue that in order to capture the intersubjective features of the case at hand (and in general), we need to bring in another level of analysis: that of social relationships. I propose a working definition for 'social relationship' and introduce the notion of social-relational emotions. I further argue that shared emotions are best understood as a special case of social-relational emotions arising within and/or giving rise to a Communal Sharing relationship.

1 According to Krueger (2013), Merleau-Ponty (1964) argues against reducing intersubjectivity to individual mental states and defends a version of JOT. I agree with the first part: it is plausible that Merleau-Ponty was not an individualist reductionist. But I strongly disagree with the second part. It makes more sense to read Merleau-Ponty as advocating the view that in order to understand intersubjectivity, we need to take the level of social relationships seriously. After all, the title of the lecture series is "The child's relations with others." A detailed exegesis of Merleau-Ponty's work is beyond the scope of this paper-I will leave it to scholars of Merleau-Ponty.

${ }^{2}$ I focus on JOT as outlined in (Krueger 2013). In some more recent papers, Krueger expresses somewhat more modest views on JOT (see e.g., Colombetti and Krueger 2015), but my focus in this paper is what I take to be the strongest version of Krueger's JOT. 


\section{Sharing mental states}

In order to provide a general background to the discussion in this paper, I briefly survey various ways the sharing of mental states has been understood in the philosophical literature.

The most common way in which sharing a mental state is understood is as sharing the same general type of a mental state by having different tokens of that type. For example, two persons can share a thought that a movie they are watching is boring or they can share an intention to have dinner. Each person has their own individual token of a mental state, e.g., a thought with the content 'this movie is boring.' Even though those states may be directed at the same real or intentional object (movie or dinner), the particular tokens of the general mental state type are strictly individual. In the special case when the shared states are dispositional, not occurrent, the type-token distinction still applies. Sharing the same preference for movies or being inclined to intend the same kinds of actions are examples of sharing dispositional states in that sense. Although both persons can be characterized in the same way at the general level of the mental state types, their individual token-dispositions would still be distinct from each other. This sense of mental state sharing is uncontroversial: no one doubts that we can share mental states with other people in this "thin" sense. But there is nothing inherently intersubjective or social about having different tokens of the same type of mental state. Authors who wish to conceive of shared mental states as inherently social have tried to locate the intersubjective element elsewhere. I will now review these other options.

In reviewing those other options, I proceed from a useful distinction between the content, ${ }^{3}$ the mode, and the subject of a mental state. ${ }^{4}$ The subject of a mental state is the one who has the state, and this can be either a single or a plural entity; e.g., an individual or a group. The content fixes the (intentional) object or state of affairs to which the state is directed. The mode specifies the way the mental attitude (e.g., believing, desiring or fearing) is directed at the object or state of affairs. The intersubjective element can be located in any of these three components of the intentional state.

One family of views finds the intersubjective element in the content of an intentional state. The crux of the idea is that the state is directed at some collective circumstance, like doing something together. The content of such a state might be, e.g., that 'we do $x$ together'. A more complex example of such a view is provided in Michael Bratman's (1999) account of shared intentions.

${ }^{3}$ I focus here on mental states that have content and leave aside mental states that lack intentional content; e.g., moods, pains or itches.

${ }^{4}$ See (Schweikard and Schmid 2013). The following brief overview in this section is based on their article. 
On his view, shared intentions consist primarily of attitudes of individuals and their interrelations and the "we" located in the content of the individual intentions is to be understood in a distributive sense. For an intention to $x$ to be shared, all parties to the cooperation must: intend that they do $x$ together, do $x$ because of such intentions, and do $x$ in conformity with those intentions. Moreover, that these conditions have been satisfied must be common knowledge to all parties.

The second group of views locates the collectivity in the particular mode of the shared mental state. As already said above, the mode of a mental state is the way in which the subject is directed at an object. In the context of collective intentionality, mode-theorists make a further distinction between an I-mode and a we-mode. The we-mode is the first-person plural mode of mental attitudes. Wilfrid Sellars' work on we-intention has paved the way for subsequent research in that direction (see, e.g., Tuomela and Miller 1988). John Searle (2010) can be seen as a mode theorist, although he himself does not use the term 'we-mode.'5 One of the most comprehensive accounts of a we-mode belongs to Raimo Tuomela (2013). Drawing on Tuomela's account, Michael Schmitz (2017) distinguishes between subject content and object content and argues that in a we-mode, other subjects are represented not as objects, but as co-subjects in the subject content. A version of the we-mode approach has been defended also from a neuroscientific point of view by Gallotti and Frith $(2013,163)$, who understand representing aspects of the world in the we-mode as "representing aspects of the interactive scene in a distinct psychological attitude of intending-together, believing-together, desiring-together."

The third option is to place the collectivity in the subject of the mental state. This means postulating plural subjects or groups as agents that are taken to be irreducible to individual members of them and to have certain mental states of their own. A version of the plural subject view is defended by Margaret Gilbert. On her view, such subjects are created by undertaking a joint commitment that implies normative obligations (Gilbert 2006).

As we will shortly see, some of Krueger's claims suggest that he gravitates towards the plural subject view. However, his main position, supported by his reading of Merleau-Ponty, seems to constitute an altogether different and very radical approach, namely the thesis that two agents can literally share the same mental state token. I will introduce Krueger's position in the next section.

${ }^{5}$ But see (Salice 2015) for a critique of Searle's we-intentions as primitive mental attitudes and a defense of the view that Searle's account reduces to a version of the content approach. 


\section{Krueger's view: Jointly owned positive emotions in early in- fancy}

Krueger $(2013,511)$ defends the joint ownership thesis (JOT): the idea that "two subjects (...) can be said simultaneously to share the same episode of emotion." He motivates his view by his reading of Merleau-Ponty's famous lecture series "The child's relations with others" (Merleau-Ponty 1964). Krueger argues that Merleau-Ponty can be interpreted as defending the view that "certain early experiences are jointly owned, in that they are numerically single experiences that are nevertheless given to more than one subject" (Krueger 2013, 509). He further claims that according to Merleau-Ponty "the newborn is experientially coupled to the caregiver in a state of phenomenal undifferentiation" (Krueger 2013, 514). He then moves on to support JOT by drawing on an analysis of the case of positive emotions in very young infants based on research in contemporary developmental psychology.

On Krueger's account, the case of positive emotions in young infants is a good candidate for a jointly owned mental state for two main reasons. Firstly, he argues that young infants are incapable of experiencing positive emotions on their own. A young infant is only able to experience them while interacting with a caregiver who is currently in a positive emotional state. The reason for this is that young infants' attention is primarily exogenousi.e., infants have not yet developed top-down control over their attention, so their attention is driven by the environment, and often by their caregivers (Krueger 2013, 515). In order to experience a positive emotion, the infant needs to attend to some "happy stimuli" for a minimal period of time to enable a positive emotion to arise. For young infants, such happy stimuli are provided by caregivers when they interact with the infants and are in positive affective states themselves. From this, Krueger concludes that in order to experience positive emotions, infants rely on the cognitive scaffolding that their caregivers offer them during social interactions. In other words, infants can have positive emotions only by forming a coupled cognitive-affective system with their caregiver (Krueger 2013, 516).

Secondly, Krueger argues, following Merleau-Ponty, that there is a lack of robust self-other differentiation in the phenomenological structure of the experiences of positive emotions in young infants. Although he is careful to point out that newborns are already likely to possess a very basic bodily sense of self, he argues that the structure of their emotional experience probably lacks stable self-other differentiation because young infants have limited inhibitory control. The lack of inhibitory control makes infants experience emotions without the opportunity to actively regulate those emotions, so that they have an attenuated sense of "emotional agency" (Krueger 2013, 518). On Krueger's account, the sense of agency is a necessary compo- 
nent of the awareness of oneself as a stable and enduring perspective on the world, distinct from others. So he argues that when infants experience positive emotions, they dwell in a state of experiential undifferentiation, more or less in the way Merleau-Ponty's phenomenological analysis arguably suggests.

\section{Why JOT does not work}

Krueger's account is highly problematic in several respects. In the following, I outline four problems with Krueger's account.

\subsection{Joint ownership or joint subjects?}

Although Krueger's (2013) goal is to outline and argue for JOT-the thesis that two or more subjects can jointly own an emotion-he also writes about joint subjects. This is confusing. For example, in parallel to the clearly stated expressions of JOT, Krueger writes that "the structure of some early infant-caregiver dyadic exchanges is best described as involving joint subjects" (Krueger 2013, 509-510, emphasis added) and that "it is sensible to speak of caregivers as entering into the infant's experience as a joint subject" (Krueger 2013, 522, emphasis added). How is the talk about "joint subjects" to be understood and is it compatible with JOT?

One interpretation of these statements is that Krueger gravitates towards the plural subject thesis - the view that two or more individuals form a higher order entity that has mental states of its own. But it remains unclear whether one can consistently hold the position that two individual subjects own the same token of a mental state and at the same time there is a single higher order subject having the mental state: it seems to lead to an overdetermination on the part of the subject(s) of the mental states.

Another possible interpretation would be that by joint subject(s) Krueger actually means an aggregate subject composed of the infant and the caregiver. Krueger's JOT would then take the form of the claim that under certain conditions, aggregate subjects can have a single token of an emotion. This view is not logically inconsistent, but it would be a somewhat odd combination of positions. The aggregate subject approach is the view that collectives are reducible to sums or aggregates of individuals and it goes hand in hand with the view that shared or collective mental states are reducible to aggregations of individual states. Among researchers of collective intentionality, this view is rather unpopular and typically criticized as too reductive and incapable of explaining human sociality (see Schweikard and Schmid 2013). At best, a combination of JOT and the aggregate subject approach would simply collapse into pure JOT and would make talking about aggregates or "joint subjects" superfluous. At worst, it would be a controversial view: JOT is clearly a 
non-reductive account in that it does not reduce shared emotions to a simple sum of two individual emotions, whereas the aggregate subject view seems to imply reductionism. So the charitable conclusion to be drawn from this is that Krueger probably does not have the aggregate subject or the plural subject view in mind when he talks about "joint subject(s)."

Yet another possible, and perhaps more plausible, interpretation would be that by "joint subjects" Krueger means something more distributive than a higher order entity, but still something more substantial than an aggregate subject. Joint subjects would be composed of individuals and their interrelations, but there would be no higher order entity ontologically speaking. This view would be consistent with JOT and might have potential. By "joint subjects" Krueger might simply mean a plurality of individuals that participate in one and the same token of an emotion. ${ }^{6}$ Unfortunately, Krueger does not explain what exactly he has in mind when he talks about joint subjects, thus I will not pursue this matter further. My main aim is to draw the reader's attention to the fact that Krueger's account remains vague in this respect.

Let me offer one more possible reading-in fact, I think this is what might be really going on. Krueger's talk about "joint subjects" may be a label for his phenomenological description of young infants' experiences of positive emotions-i.e., a label for their subjective experience of dwelling in a state of social undifferentiation. As I will explain in the next section, it is important to keep the phenomenological level of analysis apart from ontological claims about mental states. JOT is clearly an ontological claim about mental states, whereas if my interpretation is on the right track, Krueger's talk about joint subject(s) refers to the phenomenological description of infant's experience. Unfortunately, Krueger confuses these two levels of analysis and this is where he gets things wrong, as I will show in the next section.

\subsection{Lumping together phenomenological and ontological claims}

The analysis in the previous section revealed that Krueger's vacillation between the formulations of JOT and the idea of joint subjects makes most sense if we interpret them as claims on two different levels of analysis: whereas JOT is an ontological claim, Krueger's talk about joint subjects is restricted to the phenomenological analysis of infants' experience. Although phenomenological analyzes of experiences may inform and set constraints on ontological claims about mental phenomena and vice versa, phenomenological and ontological claims do not directly reduce or translate into each other, so one must be careful when moving between the two levels of analysis.

${ }^{6}$ I thank the anonymous reviewer for pointing out this option to me. 
Krueger $(2013,511)$ indeed explicitly acknowledges JOT as a matter of ontology: "This is a claim about the ontology of certain early experiences." However, in his defense of JOT, he appeals to his phenomenological analysis of the structure of experience of positive emotions in very young infants. He argues that the state of phenomenal undifferentiation-the lack of robust self-other differentiation in the structure of infant's experience- "is the phenomenological condition that makes JOT possible" (Krueger 2013, 514). This claim is perplexing. JOT is the thesis that two or more subjects own one and the same token of an emotion. But what does it mean to own an emotion? Let's take occurrent states like happiness or joy as an example. Owning a token of a joyful emotion implies experiencing it. In the context of JOT this would translate into the claim that the experience of the emotion is the same in all individuals jointly owning the emotion. In other words, having the same token of an emotion implies having the same token of experience of the emotion. Indeed, this is one way that Krueger $(2013,509)$ formulates JOT: "certain early experiences are jointly owned in that they are numerically single experiences that are nevertheless given to more than one subject" (emphasis added). However, Krueger's phenomenological analysis of the infant's experience contradicts JOT. If Krueger's phenomenological analysis is on the right track, the structure of the infant's experience is fundamentally different from the structure of the caregiver's experience. While the latter has an underlying structure of self-other differentiation, the former lacks such structure, or so Krueger argues. However, it would be strange to say that two individuals have the same token of an experience of an emotion if their experiences are differently structured.

Let us take a closer look at what JOT implies. Having the same token of an experience of an emotion could mean either:

a) That the experience of the two individuals undergoing a jointly owned emotion is qualitatively and numerically identical. So if Krueger is right about the structure of the infant's experience of positive emotions being different from the structure of the caregiver's experience, infants and caregivers cannot jointly own emotions because their experiences are structurally different and thus both qualitatively and numerically non-identical. (However, the possibility that two people who have an experience with the same qualitative structure could jointly own a single experience is still open. I will turn to this issue below and show that even if that were the case, JOT still does not follow.) 
b) That there are numerically single emotions that can be experienced differently (for example, because they have a different phenomenological structure) by different subjects.

The second option is very counter-intuitive and would go against most if not all theories of emotions. Occurrent emotions are not objects separate from how they are experienced. It is possible for two people to have different interpretations of the same emotion, but this is another matter-here we are talking about the very experience of emotion. The structure of the experience is partly what makes the emotion what it is, so it would be odd to say that two individuals can experience the same emotion while the structure of their experience is different. Thus it is reasonable to assume that (a) holds. It means that JOT applies to the case of emotions only if the structure of the experience of the infant coincides with the structure of the experience of the caregiver: either by way of the phenomenology of the infant being analogous to that of the caregiver (and Krueger being wrong about the phenomenology of infants), or by way of the adult's experience also temporarily lacking the structure of self-other differentiation. Both options seem unlikely, but let us just grant for the sake of argument that one or another is sometimes the case. It would still only take us halfway towards JOT. Individuals having an experience of an emotion with the same phenomenological structure is necessary, but not sufficient, for jointly owned emotions. Identical phenomenological structure would be a prerequisite for qualitative identity of the two mental states, but no proof of their numerical identity.

\subsection{Coupling-constitution inference}

Another line of argument that Krueger uses in favor of JOT draws on contemporary developmental psychology. It stems from the claim that very young infants are simply incapable of experiencing positive emotions without the moment-by-moment scaffolding offered by their caregivers (Krueger 2013, 519). The claim is likely to raise a few eyebrows. After all, we know that even newborns readily express negative emotions when they are hungry or distressed, so what is so different about positive emotions? What is the evidence that young infants cannot have positive emotions without the help of caregivers?

As I explained in the previous section, Krueger relies on the fact that the attention of very young infants is largely driven by the environment and often regulated by caregivers. When a caregiver interacts with an infant while being in a positive emotional state oneself, the chances are that the infant will also shift to a positive affective state. Krueger $(2013,521)$ describes this as the caregiver's positive affect "expanding" to include the infant, so that they end up jointly owning the same token of the positive emotion. 
It is plausible that because of their attentional limitations, infants cannot sustain positive emotions on their own. However, it is far from obvious that only caregivers who are genuinely experiencing positive emotions themselves can evoke and support positive emotions in young infants. If the issue comes down to providing "happy stimuli" to infants over whatever period of time is required for the emotion to arise, an actor who merely pretends to have positive emotions seems sufficient. Perhaps an interactive toy or a funny cartoon can do the job. Then the question becomes: are cases where caregivers with genuine positive affect trigger positive emotions in infants of an ontologically different type than the positive emotions that are triggered in other ways, for instance by a toy? Are the former cases examples of jointly owned mental states whereas the latter would be cases of individual mental states? After all, toys or cartoon characters do not experience emotions, so it seems that the latter cases cannot possibly be cases of jointly owned emotions.

For Krueger's purposes, it does not have to be shown that only caregivers experiencing positive emotions can support positive emotions in infants. It is sufficient, and indeed plausible, to assume that positive emotions in young infants are sometimes (perhaps most of the time) supported by caregivers' positive emotions. In any case, whether toys or cartoons can offer the necessary scaffolding for young infants to have positive emotions is an empirical issue that shall not seriously concern us here. I can only provide personal anecdotal evidence of having seen very young infants smiling and completely mesmerized by "Teletubbies." And there are cases where the infant is happy to see the caregiver, although the caregiver does not see the infant. If it is possible for infants to have positive emotions supported by toys or cartoons, Krueger would have to assume a kind of disjunctivism between two cases: (a) cases where a positive emotion in the infant is scaffolded by an artifact or another agent that does not undergo a positive emotion; (b) cases where a positive emotion in the infant is scaffolded by another subject who genuinely undergoes a positive emotion. The latter would be a case of jointly owned positive emotion, whereas the former is a priori excluded from being a jointly owned state, because the infant is the only subject undergoing any experience. It is more parsimonious and less mysterious to say that the infant has an individual experience of positive emotion in both cases. So what reasons could there be to believe otherwise?

Let us take a look at how Krueger's argument proceeds. He argues that the caregiver and the infant function as a coupled system: "While coupled with the caregiver, this scaffolding allows the infant to exceed her developmental constraints and achieve a flexibility and stability of attention well beyond her current developmental level" (Krueger 2013, 516). From the claim 
about coupling he moves on to the claim that " $(\mathrm{t})$ he emotion of the caregiver is (...) a constituent part of the infant's emotional experience (...) the caregiver's emotion is part of the very process responsible for the realization of the emotion within the infant's experience" (Krueger 2013, 521). Here, Krueger makes a coupling-constitution inference: he begins by identifying a causal relation or "coupling" between the caregiver's emotion and the infant's emotion and ends up inferring that there is a constitutive relation between the two.

The same kind of inference is often present in accounts of the extended mind (see e.g., Clark and Chalmers 1998; Menary 2010). For instance, Alva Noë $(2004,221)$ argues that "the environment can drive and so partially constitute cognitive processes." Critics of the extended mind approach argue that the coupling-constitution inference is seriously flawed; they have even labeled it the coupling-constitution fallacy (CCF) (see e.g., Adams and Aizawa 2001; Adams and Aizawa 2008; Adams and Aizawa 2010; Aizawa 2010). Adams and Aizawa (2010, 590) write:

The fallacy in this kind of argument is easy to see (...) the fact that a process $\mathrm{X}$ is coupled to a process of type $\mathrm{Y}$ does not show that that $\mathrm{X}$ is, in fact, also a (part of a) $Y$ process or that the entire $X-Y$ process is of type $\mathrm{Y}$.

In recent years, there have been heated discussions over CCF in circles of defenders and opponents of extended mind and extended cognition. The proponents of the extended mind approach can pursue one of the two following strategies for defending themselves against the charge that they have committed the CCF. One strategy is to admit that an inference from coupling to constitution is indeed fallacious, but to deny that for an extended mind account an inference from coupling to constitution is necessary (cf. Aizawa 2010, 334). This strategy is not open to Krueger because the couplingconstitution inference is clearly central to his argument. The other strategy is to show that a coupling-constitution inference is not necessarily fallacious. Several authors have argued that the CCF argument is question-begging against the hypothesis of extended cognition, because it presupposes that cognition cannot extend beyond the organism (see e.g., Menary 2006; Hurley 2010; Ross and Ladyman 2010; Kagan and Lassiter 2013). To this, Adams and Aizawa $(2010,595)$ have responded that their argument would remain unaffected even under the presupposition that an individual's "biological mass never in itself suffices to form a cognitive system."

Since Krueger clearly relies on a coupling-constitution inference, much of the debate on CCF applies to his account of JOT. Unfortunately, Krueger (2013) does not acknowledge that the coupling-constitution inference might be problematic. In a more recent paper, where he defends various versions 
of the extended emotions hypothesis, including a version of JOT, he does briefly relate to the discussion on CCF, but quickly dismisses the whole discussion as having reached "something of a stalemate" (Krueger 2014, 544). Krueger is right that there is a lack of consensus over how serious a threat CCF poses to the extended cognition approach. Although I think that Krueger's inference from coupling to constitution is unwarranted, I will not pursue the debate over CCF any further at this point. As I will show in the next section, Krueger's account of JOT falls apart even if we were to grant the coupling-constitution inference as valid.

\subsection{Confusing the system that realizes the emotion with the owner of the emotion}

Krueger's JOT goes a lot further than the classical extended mind approach. It can be seen as a very specific and radical version of the extended mind account. In what follows, I argue that Krueger's JOT fails even if we were to favor the extended mind approach in general.

The classical example of a case of extended mind is the story of Inga and Otto (Clark and Chalmers 1998). Inga is a healthy individual, whereas Otto suffers from a mild form of Alzheimer's disease and has difficulties with his memory. In order to go to the Museum of Modern Art, Inga consults her biological memory, whereas Otto consults his notebook for the address of the museum. Clark and Chalmers argue that since Otto always carries his notebook around and uses it regularly and reliably to remind him of the quotidian facts that healthy individuals typically retrieve from their biological memory, his notebook has become a part of his cognitive system just like Inga's biological memory-system is part of her cognitive system - the notebook serves the same cognitive function for Otto as Inga's memory serves for her. Krueger seems to argue in a similar fashion: since the caregiver enables the young infant to overcome his or her cognitive limitations, the system that brings about the infant's positive emotion is extended in such a manner that it includes the cognitive-affective processes of both individuals. ${ }^{7}$ As we have already seen, Krueger $(2013,521)$ states that " $(t)$ he emotion of the caregiver is thus a constituent part of the infant's emotional experience (...)." Notice, however, that this claim is at odds with JOT: if the caregiver and the infant have the same token of an emotional experience, as JOT requires, how can one be part of the other? They are supposed to be identical. ${ }^{8}$ The puzzle

7 One might argue that the two cases are disanalogous: whereas Otto is cognitively disabled, the young infant is a perfectly healthy individual. However, both have limited cognitive capacities in comparison to a healthy adult.

${ }^{8}$ In principle, it is possible that the token emotion co-owned by the individuals is a complex entity and hence has parts that belong to different people. I am grateful to the reviewer for 
indicates that JOT is a much more demanding thesis than the classical hypothesis of the extended mind: it requires more heavy lifting than reliance on a coupling-constitution inference, even if the latter were a valid inference.

The classical extended mind hypothesis concerns the vehicles of cognitive processes and non-occurrent mental states such as dispositional beliefs or memories. Clark $(2010,45)$ explicitly states that with the extended mind hypothesis he and Chalmers "allowed that (as far as our argument was concerned) conscious mental states might well turn out to supervene only on local processes inside the head" (emphasis added). In contrast, Krueger focuses on conscious occurrent mental states-experiences of emotions. Furthermore, he claims not only that the processes that realize infants' positive emotions are socially extended, but also that the resulting experiential state is jointly owned by the infant and the caregiver. He moves from the claim that "the caregiver provides socio-cognitive resources (positive affect) and capacities (endogenous attention) that the infant incorporates into her own basic suite of sensorimotor skills to (jointly) realize the experience" (Krueger $2013,523)$ to the claim that "infant and caregiver might thus be said to share the same episode of emotion" (Krueger 2013, 524). Unfortunately, he jumps without argument from the claim

a) the extended infant-caregiver system realizes the emotion of the infant

to the claim

b) the infant and the caregiver jointly own the same token of the experience of the emotion.

In fact, he does not seem to notice that these are two different claims and that an extra argument is needed to make the latter point.

It is hard to see what argument could be put forward in favor of (b). Even if it would make sense to say that positive emotions in young infants are realized not only by their brains, but by a socially extended system, it

pointing out this possibility. But I find it hard to make sense of the idea that the infant and the caregiver experience the same token of an emotion (which might very well be a complex entity) and, at the same time, that one person's emotion is a part of the jointly owned emotion. What does the individual experience then-her own individual emotion as a part of the jointly owned emotion or the whole complex entity or both? If two individuals experience only their respective "parts" of the jointly owned emotion, then in what sense do they have the same experience? How many emotions are involved in the joint experience, ontologically speaking: one, two, or three? Perhaps it would make more sense to say that different individuals can experience different aspects of the same shared emotion-Schmid has been arguing in this direction (see Schmid 2009, 82). 
would not follow that a single emotion is owned or experienced by two subjects. Usually, the cognitive system that realizes some mental state and the owner/experiencer of the state do not coincide: the system is described at the sub-personal level, whereas the owner of the state is the whole organism or person. To illustrate: in humans, a particular activation of the insula realizes the experience of disgust, but it is not the case that the person and the insula jointly own the disgust experience. The insula does not experience anything.

Another line of thought against JOT emerges from the following analogy between positive emotions in infants and a common experience of tickling. It is well known that healthy individuals cannot tickle themselves. I only have the experience of being tickled when another person tickles me. This has to do with a certain "limitation" of how my brain works: I cannot prevent the brain from predicting what self-touch feels like and therefore tickling oneself does not feel tickly (see Blakemore et al. 1998). The "limitation" can be easily overcome: all I need is someone else to tickle me. To use the jargon of the extended mind approach: the system that realizes my experience of being tickled is an extended system that includes the activity of my tickler. But this would not make my experience of being tickled a jointly owned experience: the experience of being tickled is owned by just one subjectme.

The analogy between the case of tickling and the positive emotions in infants is not perfect. In the latter case, both the caregiver and the infant end up experiencing a positive emotion. Does that provide a good reason to think that the infant and the caregiver experience the same episode of emotion? I do not think so. Let us expand the example of tickling into a little thought experiment. Imagine a world where there are two types of people: those who can tickle themselves and those who need others to tickle them to have the experience of being tickled. ${ }^{9}$ Tim is of the first type, whereas Nora is of the second. Now imagine that Tim tickles himself and then begins to tickle Nora. They end up tickling each other, so that both have an experience of being tickled. Does it mean that they end up having the same token of an experience of being tickled? Obviously not. The tickler case is analogous to the case of positive emotions in young infants: the caregiver approaches the infant while being in a positive emotional state, helps the infant to achieve a positive emotional state, and they end up sustaining the positive emotion in each-other via mutual interaction. However, the infant and the caregiver have individual tokens of the experience of positive emotion.

9 In fact, this could be the world we currently live in: there is evidence that patients with certain psychiatric disorders like schizophrenia may be able to tickle themselves (see Shergill et al. 2005). 


\subsection{Summary of the critique of JOT}

Joel Krueger analyzes the case of positive emotions in young infants and argues that it is an example of two individuals jointly owning the same token of an occurrent mental state. Krueger's account is flawed in many respects. Firstly, although his aim is to defend JOT, he also talks about joint subjects without specifying what he means, which is more confusing than helpful. Secondly, Krueger lumps together phenomenological and ontological claims. Upon closer inspection, his phenomenological analysis of the experience of the infant actually speaks against JOT as an ontological claim. Thirdly, Krueger relies on a coupling-constitution inference that is controversial, to say the least. Fourthly, he makes an illicit move from the claim that the infant-caregiver system realizes the positive emotion of the infant to the claim that the two subject jointly own the experience. This leads us to the general conclusion that JOT fails as an account of shared mental states.

\section{Positive proposal: Shared emotions are a special type of social- relational emotions}

Although JOT is false, there does seem to be something inherently social or intersubjective in the experience of positive emotions in young infants. How can we make sense of this intersubjective dimension without postulating mysterious mental entities owned by more than one individual, but do so without reducing intersubjectivity to just a sum of strictly individual mental states? I will outline an alternative way of thinking about shared emotions by shifting to another level of analysis: that of social relationships. In what follows, I argue that most cases of what researchers call 'shared emotions' fall under a subtype of social-relational emotions: emotions that arise out of social relationships, and whose focus is some aspect of the relationship. I will then apply this new approach to the case of positive emotions in young infants.

\subsection{What are social relationships?}

Before I can introduce the idea of social-relational emotions, I first need to explain what I mean by social relationships. What is a social relationship? Simply put, a social relationship is an inherently intersubjective phenomenon: it does not reside within an individual mind, but can instead be described as "the oscillating rhythm of influence observed in the interactions of two people" (Berscheid 2004, 32). An increasingly common way to analyze social relationships is to model them as self-organizing dynamical systems (Butler 2011). Relationships as self-organizing systems are seen as organizing ensembles composed of closely integrated elements. Past states 
of the system partially influence its future states and temporal changes of the system are analyzable as functions of interactions among the elements. According to the dynamical systems approach, social relationships emerge as higher order patterns of interactions that partially constrain and coordinate the lower level elements (Butler 2011, 368).

At the same time, social relationships also have a cognitive dimension: as humans, we do not just form relationships with others, we also cognitively represent features of relationships that are not features of the participating individuals as such (Fiske and Haslam 1996). According to relational models theory, proposed by cognitive anthropologist Alan Page Fiske, coordination of human social relationships is based on combinations of four elementary cognitive models of social relationships (Fiske 1991; Fiske 1992). The four elementary structures describe both social relationships in the world as well as people's implicit understanding of relationships. In other words, relational models capture both psychological as well as ontological features of social relations.

The first model is called Communal Sharing. It characterizes relationships where people treat each other as belonging to the same social category by focusing on their commonalities and disregarding distinct individual identities. Participants are treated as equivalent in some essential respect. The model typically organizes intense romantic love, the cohesion of members of a team, as well as larger scale ethnic, national, gender, or species identification. For example, a couple in love or a group of people having the same politics or fans of the same soccer team can form a Communal Sharing relationship based on their commonalities. People in a Communal Sharing relationship experience a sense of oneness with other members of the group.

The next model, Authority Ranking, orders people into "higher" or "lower" positions in a legitimate social hierarchy. The model is clearly operative in various dominance hierarchies, such as in the military, but also in boss-employee, teacher-student, parent-child and other such relationships where subordinates owe deference and respect, while superiors are expected to provide leadership, guidance, wisdom, and protection. Authority Ranking relationships may involve coercion and exploitation, but power abuse is not inherent to the model and renders the relationship unstable.

The third model is labeled Equality Matching. As the label suggests, it embodies a principle of equality. People treat each other as individuals with an equal status, and seek for balance in interactions, as is often the case in interactions among colleagues, equal partners, friends or sometimes enemies. Interactions within Equality Matching relationships may take the form of turn-taking, balanced in-kind reciprocity, or eye-for-an-eye vengeance. 
Finally, in Market Pricing, social interactions are coordinated with respect to socially meaningful ratios, rates, or proportions. Market Pricing underlies utilitarian moral reasoning, proportional justice, cost-benefit social decision making, as well as prices, rents, wages, academic grading, etc.

According to the relational models theory, people implicitly rely on these four cognitive models to generate, interpret, coordinate, and evaluate their social relationships. ${ }^{10}$ The theory also says that people have an inherent motivation to pursue relationships for their own sake and not just for external reasons. People use relational models with reference to cultural knowledge that specifies how, when, where, and with whom a particular model should operate. Social relational differences among cultures and individuals can be explained as differences in implementation of the four models. Social conflicts arise when people apply different models to the same social situation or when they implement the same model differently. The theory has been supported by many experimental and observational studies covering populations from many cultures. ${ }^{11}$

By integrating the dynamical systems approach with the relational models approach, I define social relationships as temporally extended and coordinated complementarities of actions and/or mental states among two or more participants (cf. Bohl 2015, 681). Coordination means that participants act, think, and/or feel with reference to each other's actions, thoughts, and/or feelings. Complementarity means that what one agent is doing, thinking, or feeling is incomplete or impossible unless and until another agent meaningfully complements it.

\subsection{Social-relational emotions}

In philosophy of mind and cognitive psychology, emotions are typically defined as intrapersonal states that have specific experiential, cognitive, behavioral and physiological subcomponents. However, human emotions often arise in social contexts and have important social functions (Manstead 2006; Berscheid and Ammazzalorso 2003; Clark et al. 2003; Keltner and Haidt 1999). Relationship scientists view emotions as adaptations for dealing

${ }^{10}$ Each relational model also corresponds to a particular mathematical structure. The mathematical structure of Communal Sharing is an equivalence relation, homologous to that of a nominal scale of measurement. The structure of Authority Ranking is linearly ordered and it corresponds to an ordinal scale of measurement. The mathematical structure of Equality Matching is that of an ordered Abelian group, and corresponds to the structure of an interval scale. The structure of Market Pricing is homologous to a ratio scale and corresponds to an Archimedean ordered field. (See Fiske 1992).

${ }^{11}$ See the online bibliography that lists works applying, critiquing, or developing relational models theory: http://www.sscnet.ucla.edu/anthro/faculty/fiske/RM_PDFs/RM_ bibliography.htm (visited 22.08.2016). 
with problems related to coordination of social relationships (Butler 2011, 369; Fiske 2002). In social contexts, the subcomponents of an emotion may interact not only intrapersonally, but also across two or more people. I will call such episodes of emotions social-relational emotions. ${ }^{12}$

There are many ways in which social-relational emotions may come into existence. For example, a paradigmatic case of emotional contagion is a social-relational emotion where an affective state carries over from $\mathrm{A}$ to $\mathrm{B}$, though the affective state of A may remain uninfluenced by $B$. There are also cases where the affective states of A and B synchronously alter because of a third variable: for instance, because two people are watching the same movie or witnessing the same event. According to my definition, this would not be a genuine case of a social-relational emotion, unless there is also uni- or bidirectional influence between $\mathrm{A}$ and $\mathrm{B}$, because each person would have the same affective dynamics alone.

From the perspective of social cognition research, the most interesting cases of social-relational emotions are those where mutual influence among the affective dynamics of two or more individuals can be observed. A case in point would be two people yelling at each other, with their level of anger rising in synchrony. Another example would be one person's anger and another person's fear mutually reinforcing each other. In the jargon of extended and enactive approaches to cognition, these would be cases of coupling between the emotional subcomponents of interaction partners. However, most people would not readily call such examples cases of shared emotions. In the next subsection, I will shed some light on how we could understand shared emotions in this framework.

\subsection{Shared emotions as a special case of social-relational emo- tions}

I propose to conceptualize shared emotions as a special case of socialrelational emotions. As I explained above, not all cases of social-relational emotions are such that we would readily call them 'shared emotions.' For example, when two individuals mutually reinforce each other's irritation, we would not say that they are sharing an emotion. Shared emotions seem to be characterized by a specific feature that could be called a sense of oneness. To explain what I mean by it, I borrow from Helm (2008) a useful distinction between the subject, the target and the focus of an emotion (see also Schmid 2009, 64-65). The subject is the one who experiences the emotion. The target is something towards which the emotion is directed. For example, if

${ }^{12}$ The idea of social-relational emotions is in accordance with the temporal interpersonal emotion systems (TIES) approach, see (Butler 2011). The latter approach can be seen as a dynamical systems framework for analyzing social-relational emotions. 
I am afraid of flying then I am the subject of the fear of flying and the target of the fear is flying. The focus, however, is something in the background of the emotion that is rationally connected to the target, making the particular mode of the emotion comprehensible. In the case of fear of flying, the focus might be myself or it might be someone else; e.g., I might be concerned about the safety of my child as she travels by airplane. The subject has to have some concern that explains the connection between the target and the focus: I need to care about myself or about my child in order to feel frightened about my flying or the flying of my child.

With these conceptual tools at hand, I propose to analyze shared emotions as social-relational emotions that have a social relationship as its focus. If lovers are happy to see each other, we have two tokens of happiness with two different subjects and two different targets: I am happy to see you whereas you are happy to see me. But the focus of our happiness is the relationship between us: we are happy to see each-other, because our meeting positively feeds into our relationship. This is what I mean by sense of oneness: it is a phenomenological quality of an affective experience that arises when the focus of an affective experience of two or more individuals is their relationship in such a way that the emotion is experienced as having the quality of nourishing the relationship between the participants of the social interaction. The sense of oneness is clearly lacking when two people reinforce each-other's negative emotions in a conflict situation: although there are social-relational emotions at play, the focus of an emotion tends to be an isolated individual: I am angry at you (the target), because I experience you as misbehaving towards me (the focus). The structure of the focus might also be two-fold: I am angry at you (the target), because I experience you as misbehaving towards $m e$ (focus 1 ) and as jeopardizing our relationship (focus 2).

What are the necessary and sufficient conditions for an emotion to be shared? Do all parties need to have the relationship as the focus of their emotion for an emotion to be shared? Do all parties need to have an emotion with the same mode? What about the target? I think that the answers to these question depend on one's theoretical aims and preferences (see also Michael 2016) and I will not argue for a particular view here. Rather than a label for a particular phenomenon with a certain essence, everyday talk about sharing emotions seems to be a folk psychological construct referring to complex and diverse phenomena. Instead of arguing for a fixed set of conditions under which emotions are truly shared, a more fruitful approach might be to map out all possible social-relational emotions to create a taxonomy for describing various kinds of real life social-relational emotional experiences. For example, it seems useful to distinguish between the sub- 
jective experience of sharing an emotion and intersubjectively sharing an emotion. If I experience a sense of oneness with you whereas you lack such an experience, presumably because our relationship is not at the focus of your emotional experience, then I have a subjective experience of sharing an emotion with you without the emotion being intersubjectively shared. On the other hand, you and I might experience a strong sense of oneness even if the targets and the modes of our emotional experiences differ. For example, I might be immensely grateful to you because you rescued me from a burning house whereas you are immensely relieved that you managed to save my life, but the focus of both of our emotions might be our Communal Sharing relationship and thus a strong sense of oneness might accompany experiences of both of us. Whether we should reserve the label 'shared emotion' to one or several of these combinations of subjects, targets, focuses and modes of emotion seems more of a matter of taste than a serious theoretical issue. That being said, it does not mean that the everyday talk about sharing emotions is completely arbitrary. I would argue that a sense of oneness is at the core of sharing emotions, but it is a matter of debate whether other conditions have to be met as well and if so, what those conditions are.

Let me return to the example of positive emotions in very young infants. There are two specific moments that seem to call for an explanation: the caregiver's central role and the alleged lack of self-other differentiation in the infant's experience. We can make sense of these features by sticking to individual social-relational emotions and positing a Communal Sharing relationship between the infant and the caregiver as the focus of their respective emotions.

According to the definition given above of 'social relationship', the action or experience of one party of a relationship is incomplete or impossible unless it is complemented by another party of a relationship. This is clearly the case in Krueger's example of positive emotions in young infants, since such states arguably only arise while infants interact with caregivers. The possible lack of self-other differentiation refers specifically to a Communal Sharing relationship. Such relationships are based on the common supra-individual identity and not on separate individual identities. However, we should take Krueger's phenomenological analysis with a grain of salt, because contemporary developmental psychologists would argue that newborns already have a fundamental sense of self and it is doubtful that it magically disappears in episodes of positive emotions (see Rochat and Striano 2000). But one can, of course, also have a Communal Sharing relationship with a fully developed sense of self. The point is rather that without a self-other differentiation, it would be impossible to have other types of social relationship besides Com- 
munal Sharing relationships (e.g., Authority Ranking or Equality Matching relationships).

How do social-relational emotions arise in the case of positive emotions in young infants? The first stage can be described in terms of uni-directional influence from the caregiver to the infant: the caregiver's positive emotional state alters the emotional state of the infant in such a way that the latter matches the former at least in its positive valence and possibly also in its intensity. In the second stage, a feedback-loop within the infant-caregiver system is created: not only is the positive emotion of the caregiver influencing the affective state of the infant, but the positive emotion of the infant is in turn influencing the affective state of the caregiver. In other words, the emotional processes of two individuals become coupled. This would be a scenario most likely to be found in a Communal Sharing relationship: the infant and the caregiver become experientially united in the same type of positive emotional experience. This does not mean, however, that the two individuals own the same token of an emotion: ontologically, there are two distinct emotional states, albeit mutually dependent and synchronous. On a more fine-grained level of analysis the emotions of two individuals are clearly different; they have different subjects and targets. However, the phenomenology of both individuals may be best described as characterized by a sense of oneness, which is a typical feature of close Communal Sharing relationships and which can be explained by locating the relationship at the focus of the emotion.

Notice that social-relational emotions arise in the context of social relationships, but at the same time they also feed into relationships. If relationships are temporally extended and coordinated complementarities of actions and/or mental components among two or more participants, then social-relational emotions are important parts of the "stuff" relationships are made of. The so-called shared emotions are most likely to be found in Communal Sharing relationships: both in the sense that they more readily arise in such relationships, but also in the sense that they enable us to create, strengthen and maintain Communal Sharing relationships. Whether different types of social-relational emotions converge around different relational models is an interesting topic for future research. In the future, I hope to work out a taxonomy that categorizes social-relational emotions into different clusters, based on their structural properties.

A general point to be taken away from this analysis is that in order to understand social cognitive and affective phenomena, it is not sufficient to do research on the level of individuals and their mental states: we also need to bring social relationships into the picture. 


\section{Conclusion}

I have critically analyzed Joel Krueger's Joint Ownership Thesis: the view that two or more people can literally share the same episode of an emotion. My analysis revealed four serious flaws of Krueger's account of JOT. Firstly, Krueger fails to clearly distinguish between JOT and the thesis of joint subjects and does not explain what he means by the latter. Secondly, he confuses phenomenological and ontological levels of analysis. On a closer look, his phenomenological analysis of JOT contradicts JOT as an ontological claim. Thirdly, JOT relies on a highly problematic inference from coupling to constitution. Finally, Krueger shifts from the claim that the child and the caregiver jointly realize an emotion to a claim about joint ownership; this is a non sequitur. Although JOT is false, the case of positive emotions in young infants is still an interesting case that calls for explanation as an intersubjective phenomenon. I have argued that the best way to do this is to introduce another level of analysis: that of social relationships. I have proposed that we consider the so-called 'shared emotions' as special cases of social-relational emotions, typically arising within and/or giving rise to Communal Sharing relationships.

\section{Acknowledgments}

I am extremely grateful to Bruno Mölder for stimulating discussions and detailed feedback on earlier versions of this paper. I would also like to thank the audiences of the conferences "Reciprocity and social cognition" (23-25 March 2015, Berlin) and ESPP 2015 (14-17 July 2015, Tartu) for comments and questions. In particular, I am grateful to Pierre Jacob and Stephen Butterfill for their helpful comments. This research has been supported by the Estonian Science Foundation grant ETF9117, the Centre of Excellence in Estonian Studies (European Union, European Regional Development Fund) and is related to research project IUT2O-5 (Estonian Ministry of Education and Research).

\section{Bibliography}

Adams, F. R. and Aizawa, K. (2001). The bounds of cognition, Philosophical Psychology 14: 43-64.

Adams, F. R. and Aizawa, K. (2008). The Bounds of Cognition, Blackwell, Malden. 
Adams, F. R. and Aizawa, K. (2010). The value of cognitivism in thinking about extended cognition, Phenomenology and the Cognitive Sciences 9: $579-603$.

Aizawa, K. (2010). The coupling-constitution fallacy revisited, Cognitive Systems Research 11: 332-342.

Berscheid, E. (2004). The greening of relationship science, in H. Reis and C. Rusbult (eds), Close Relationships: Key Readings, Psychology Press, New York, pp. 30-41.

Berscheid, E. and Ammazzalorso, H. (2003). Emotional experience in close relationships, in G. J. I. Fletcher and M. S. Clark (eds), Blackwell Handbook of Social Psychology: Interpersonal Processes, Blackwell Publishing, Malden, pp. 308-330.

Blakemore, S.-J., Wolpert, D. M. and Frith, C. D. (1998). Central cancellation of self-produced tickle sensation, Nature Neuroscience 1: 635-640.

Bohl, V. (2015). We read minds to shape relationships, Philosophical Psychology 28: 674-694.

Bratman, M. (1999). Faces of Intention: Selected Essays on Intention and Agency, Cambridge University Press, Cambridge.

Butler, E. A. (2011). Temporal interpersonal emotion systems: The "TIES" that form relationships, Personality and Social Psychology Review 15: 367393.

Clark, A. (2010). Memento's revenge: The extended mind, extended, in R. Menary (ed.), The Extended Mind, The MIT Press, Cambridge, MA, pp. 43-66.

Clark, A. and Chalmers, D. (1998). The extended mind, Analysis 58: 7-19.

Clark, M., Fitness, J. and Brisette, I. (2003). Understanding people's perceptions of relationships is crucial to understanding their emotional lives, in G. J. I. Fletcher and M. S. Clark (eds), Blackwell Handbook of Social Psychology: Interpersonal Processes, Blackwell Publishing, Malden, pp. 308330.

Colombetti, G. and Krueger, J. (2015). Scaffoldings of the affective mind, Philosophical Psychology 28: 1157-1176.

Fiske, A. P. (1991). Structures of Social Life: The Four Elementary Forms of Human Relations: Communal Sharing, Authority Ranking, Equality Matching, Market Pricing, Collier Macmillan, New York.

Fiske, A. P. (1992). The four elementary forms of sociality: Framework for a unified theory of social relations, Psychological Review 99: 689-723. 
Fiske, A. P. (2002). Moral emotions provide the self-control needed to sustain social relationships, Self and Identity 1: 169-175.

Fiske, A. P. and Haslam, N. (1996). Social cognition is thinking about relationships, Current Directions in Psychological Science 5: 143-148.

Gallotti, M. and Frith, C. (2013). Social cognition in the we-mode, Trends in Cognitive Sciences 17: 160-165.

Gilbert, M. (2006). A Theory of Political Obligation, Oxford University Press, Oxford.

Helm, B. W. (2008). Plural agents, Noûs 42: 17-49.

Hurley, S. (2010). Varieties of externalism, in R. Menary (ed.), The Extended Mind, The MIT Press, Cambridge, MA, pp. 101-154.

Kagan, A. and Lassiter, C. (2013). The coupling-constitution fallacy: Much ado about nothing, Pragmatics \& Cognition 21: 178-192.

Keltner, D. and Haidt, J. (1999). Social functions of emotions at four levels of analysis, Cognition and Emotion 13: 505-521.

Krueger, J. (2013). Merleau-Ponty on shared emotions and the joint ownership thesis, Continental Philosophy Review 46: 509-531.

Krueger, J. (2014). Varieties of extended emotions, Phenomenology and the Cognitive Sciences 13: 533-555.

Manstead, A. S. R. (2006). The social nature of emotion and the emotional nature of the social, in P. A. M. van Lange (ed.), Bridging Social Psychology: Benefits of Transdisciplinary Approaches, Lawrence Erlbaum, Mahwah, pp. 213-218.

Menary, R. (2006). Attacking the bounds of cognition, Philosophical Psychology 19: 329-344.

Menary, R. (ed.) (2010). The Extended Mind, The MIT Press, Cambridge, MA.

Merleau-Ponty, M. (1964). The child's relations with others, in J. Edie (ed.), The Primacy of Perception, Northwestern University Press, Evanston, pp. 96-155.

Michael, J. (2016). What are shared emotions (for)?, Frontiers in Psychology 7.

Noë, A. (2004). Action in Perception, The MIT Press, Cambridge, MA.

Rochat, P. and Striano, T. (200o). Perceived self in infancy, Infant Behavior \& Development 23: 513-530. 
Ross, D. and Ladyman, J. (2010). The alleged coupling-constitution fallacy and the mature sciences, in R. Menary (ed.), The Extended Mind, The MIT Press, Cambridge, MA, pp. 155-166.

Salice, A. (2015). There are no primitive we-intentions, Review of Philosophy and Psychology 6: 695-715.

Schmid, H. B. (2009). Shared feelings, Plural Action, Springer, Dordrecht, pp. 59-83.

Schmitz, M. (2017). What is a mode account of collective intentionality?, in G. Preyer and G. Peter (eds), Social Ontology and Collective Intentionality, Springer, Cham. forthcoming.

Schweikard, D. P. and Schmid, H. B. (2013). Collective intentionality, in E. N. Zalta (ed.), Stanford Encyclopedia of Philosophy, Summer 2013 Edition.

URL:http://plato.stanford.edu/archives/sum2o13/entries/collectiveintentionality

Searle, J. (2010). Making the Social World: The Structure of Human Civilization, Oxford University Press, Oxford.

Shergill, S. S., Samson, G., Bays, P. M., Frith, C. D. and Wolpert, D. M. (2005). Evidence for sensory prediction deficits in schizophrenia, The American Journal of Psychology 162: 2384-2386.

Tuomela, R. (2013). Social Ontology: Collective Intentionality and Group Agents, Oxford University Press, New York.

Tuomela, R. and Miller, K. (1988). We-intentions, Philosophical Studies 53: $367-389$. 